\title{
MicroRNA-100 regulates SW620 colorectal cancer cell proliferation and invasion by targeting RAP1B
}

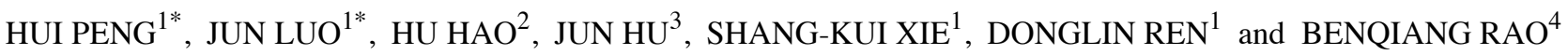 \\ Departments of ${ }^{1}$ Colorectal and Anal Surgery, ${ }^{2}$ Pediatrics, The Sixth Affiliated Hospital of Sun Yat-Sen University; \\ ${ }^{3}$ Guangdong Gastrointestinal Institute, The Sixth Affiliated Hospital of Sun Yat-Sen University, Guangzhou 510655; \\ ${ }^{4}$ Department of Gastrointestinal Anal Surgery and Institute of Gastroenterology, The Third Affiliated \\ Hospital of Nanchang University, Nanchang 330008, P.R. China
}

Received November 27, 2013; Accepted January 29, 2014

DOI: $10.3892 /$ or.2014.3075

\begin{abstract}
MicroRNAs (miRNAs) have been demonstrated to play important roles in tumorigenesis of human cancer. Fewer studies have explored the roles of miR-100 on human colorectal cancer cell proliferation and invasion. In this study, we utilized real-time PCR to verify whether miR-100 was downregulated in human colorectal cancer tissues compared with matched adjacent normal tissues. Functional studies demonstrated that ectopic expression of miR-100 inhabits cell growth and invasion and induce apoptosis, whereas knockdown of miR-100 yielded the reverse phenotype. Mechanistic studies reveal that miR-100 repressed the activity of a reporter gene fused to the 3'-untranslated region (3'-UTR) of RAP1B, whereas miR-100 silencing upregulated the expression of the reporter gene. Furthermore, we also detected that RAP1B mRNA was inversely expressed with miR-100 in colorectal cancer tissues. These data indicate that the miR-100 plays a tumor suppressor role by regulating colorectal cancer cell growth and invasion phenotype, and could serve as a potential maker for colorectal cancer therapy.
\end{abstract}

\section{Introduction}

Colorectal cancer is one of the major causes for cancerassociated death in males and females (1). Advancements in treatments involving a combination of surgical resection, radiation and chemotherapy have increased the patient's five-year survival, however, colorectal cancer remains a major public health concern (2). Therefore, an improved therapeutic

Correspondence to: Professor Benqiang Rao, Department of Gastrointestinal Anal Surgery and Institute of Gastroenterology, The Third Affiliated Hospital of Nanchang University, Nanchang 330008, P.R. China

E-mail: raobenqiang1977@163.com

*Contributed equally

Key words: miR-100, RAP1B, cell growth, cell invasion, colorectal cancer strategy is greatly needed. To our knowledge, the molecular mechanisms of colorectal cancer is complicated and still poorly understood. Although tumor-suppressor genes and oncogenes such as APC, TP53 and K-ras $(3,4)$, have been demonstrated to contribute to colorectal cancer development, only a few miRNAs have been studied to determine their roles in colorectal carcinogenesis, such as miR-21 and $\operatorname{miR}-145(5,6)$.

MicroRNAs (miRNAs) are a broad class of small, noncoding endogenous single RNA molecules that play important roles in gene expression through directly binding to the 3'-untranslated region (3'-UTR) of target gene mRNA, leading to mRNA cleavage or translational repression (7). They are differentially expressed in human cancers and play essential roles in carcinogenesis. For instance, number of miRNAs dysregulated in colorectal cancer were revealed by microarray profiles in colorectal cancer tissues compared to normal tissues, including miR-100 (8). However, there are fewer studies on the roles of miR-100 in colorectal cancer.

In this study, we identified a new anti-proliferative, proapoptosis and anti-metastatic miRNA, miR-100, in colorectal cancer cells that is frequently downregulated in colorectal cancer tissues compared to normal tissues. Accordingly, ectopic expression of miR-100 could inhibit SW620 cell proliferation and invasion, while blockage of miR-100 yielded the reverse phenotype. Moreover, we identified RAP1B, a putative oncogene in colorectal cancer, as the direct functional target of miR-100.

\section{Materials and methods}

Ethics statement and human colorectal carcinoma tissues. All specimens were from patients who underwent surgery at the third affiliated hospital of Nanchang University Hospital. The protocol had the approval of the Clinical Research Ethics Committee of Nanchang University School of Medicine, and the research was carried out according to the provisions of the Helsinki Declaration of 1975. Written informed consent was obtained from all participants involved in the study.

Cell lines and transfection. The colorectal carcinoma cell line SW620 was purchased from the Cell Bank of Type Culture 
Collection of Chinese Academy of Sciences, Shanghai Institute of Cell Biology, Chinese Academy of Sciences. SW620 cells were maintained in RPMI-1640 containing 10\% fetal calf serum. Cultures were incubated at $37^{\circ} \mathrm{C}$ in standard tissue culture incubators. MiR-100 mimics, miR-100 inhibitor (anti-miR-100) were synthesized by Genepharma, Shanghai, China. Oligonucleotide transfection was performed with Lipofectamine 2000 reagents (Invitrogen, Carlsbad,CA, USA). The final concentration of miR-100 mimics or anti-miR-100 in the transfection system was $100 \mathrm{nM}$. Transfection efficiency for the single and co-transfected studies was determined by fluorescence microscope.

RNA extraction and real-time PCR. Total RNA was extracted using TRIzol reagent. Real-time PCR analyses were carried out to detect mRNA expression using SYBR Premix Ex Taq (Takara, Dalian, China), and GAPDH was used as an internal control. MiRcute miRNA qPCR detection kit (Tiangen, Beijing, China) was used to quantitate the expression levels of mature miR-100 according to the provided protocol, and U6 was used as an internal control.

Open access software. Targetscan was used to predict the putative targets of miR-100 (http://www.targetscan.org/). miRNA-Map 2.0 was used to ananlysis the expression of miR-100 in colon cancer tissues and normal colon tissues (http://mirnamap.mbc.nctu.edu.tw/).

Cell proliferation and colony formation assay. A cell proliferation assay was performed with the Cell Counting Kit-8 (Dojindo, Kumamoto, Japan) according to the manufacturer's instruction. For the colony formation assay, 1000 cells were placed in each $100-\mathrm{mm}$ cell culture dish and maintained in media containing $10 \%$ FBS for two weeks. Colonies were fixed with $4 \%$ paraformaldehyde and stained with coomassie brilliant blue.

Annexin V-FITC and PI staining. Enumeration of apoptotic cells was done by using FITC conjugated Annexin V and PI (BD Pharmingen, San Jose, CA). Cells were washed twice in cold $1 \mathrm{X}$ PBS and resuspended in Annexin V-binding buffer (BD Pharmingen) at a concentration of $3 \times 10^{6}$ per ml. This suspension $(100 \mu \mathrm{l})$ was stained with $5 \mu \mathrm{l}$ of Annexin V-FITC and $5 \mu \mathrm{l}$ PI. These cells were gently vortexed and incubated for $15 \mathrm{~min}$ at room temperature in the dark. After addition of $400 \mu \mathrm{l}$ of binding buffer to each tube, cells were analyzed by flow cytometry.

Cell invasion assay. For invasion assay, the membrane invasion culture system (Transwell membranes of $6.5-\mathrm{mm}$ diameter and $8-\mathrm{ml}$ pore size; Costar) was used according to the standard protocol. Briefly, Harvested cells $\left(1 \times 10^{5}\right)$ suspended in $100 \mu \mathrm{l}$ of serum free RPMI-1640 were added into the upper compartment of the chamber. A total of $1000 \mu \mathrm{l}$ conditioned RPMI-1640 medium with $20 \%$ (v/v) fetal bovine serum was used as a source of chemoattractant and placed in the lower compartment of the chamber. After $48 \mathrm{~h}$, the non-invasive cells on the upper surface of the membrane were removed with a cotton swab. The transformed cells that migrated through the Matrigel matrix and stuck to the lower surface of the membrane were fixed with $4 \%$ paraformaldehyde, stained with $1 \%$ crystal purple. The invasive cells were then counted (five high-power fields/chamber) using an inverted microscope. Each test was repeated in triplicate.

Luciferase assay. SW620 cells were cultured in 24-well plates and cotransfected with $100 \mathrm{nM}$ of miR-100 mimics or antimiR-100, 100 ng reporters, and $10 \mathrm{ng}$ pGL3-CMV Renilla luciferase reporter using Lipofectamine 2000. After $24 \mathrm{~h}$ of transfection, firefly and Renilla luciferase activities were measured using the dual-luciferase reporter assay system (Promega, Madison, WI, USA).

Western blotting. Proteins were separated on 15\% SDS-PAGE gel and then transferred to PVDF membrane. After blocked with 5\% nonfat milk, the membrane was incubated with rabbit anti-RAP1B polyclonal antibody (1:1000, Proteintech, Chicago, IL, USA) and anti-GAPDH antibody (Abcam, San Francisco, CA, USA, 1:1000 dilution). The secondary antibody was goat anti-rabbit IgG conjugated with HRP (horseradish Peroxidase) with a dilution of 1:1000. The bound antibodies were detected using ECL Plus Western blotting detection system (GE Healthcare). GAPDH was used as an internal control to normalize RAP1B expression level.

Tumor formation assay in a nude mouse model. The SW620 cells $\left(5 \times 10^{6}\right)$ were injected into the flanks of athymic nude mice. One week after the injections, mice with comparably sized tumors were treated for 4 weeks with miR-NC and miR-100 mimics. Tumor growth was examined twice a week. After 4 weeks, the mice were sacrificed and examined for the growth of subcutaneous tumors.

\section{Results}

miR-100 is downregulated in human colorectal cancer specimen. MiRNA-Map-2.0 was used to investigate miR-100 expression in normal colon compared with tumor colon. As shown in Fig. 1A we found that miR-100 levels were frequently downregulated in tumor human colon compared to normal colon. To explore the possible role of miR-100 in human colorectal cancer development, we detected miR-100 expression in human colorectal cancer specimen obtained from 8 patients by real-time PCR. The carcinoma tissues showed reduced miR-100 expression with respect to normal counterparts, which is consistent with the miRNAMap-2.0 gene chip results (Fig. 1B). Together, these results suggest that miR-100 plays an important role in colorectal cancer development.

miR-100 regulates proliferation of human colorectal cancer SW620 cells in vitro and in vivo. To determine the role of miR-100 in proliferation of colorectal cancer cells in vitro, MTT assays and colony formation assays were performed. Real-time PCR results showed the miR-100 mimics could significant increased the endogenous miR-100 expression level while anti-miR-100 mimics greatly reduced its expression in SW620 as showed in Fig. 2A. Then we utilized the miR-100 mimics and anti-miR-100 mimics compared with their relative control mimics to infect the SW620 cells. MTT 


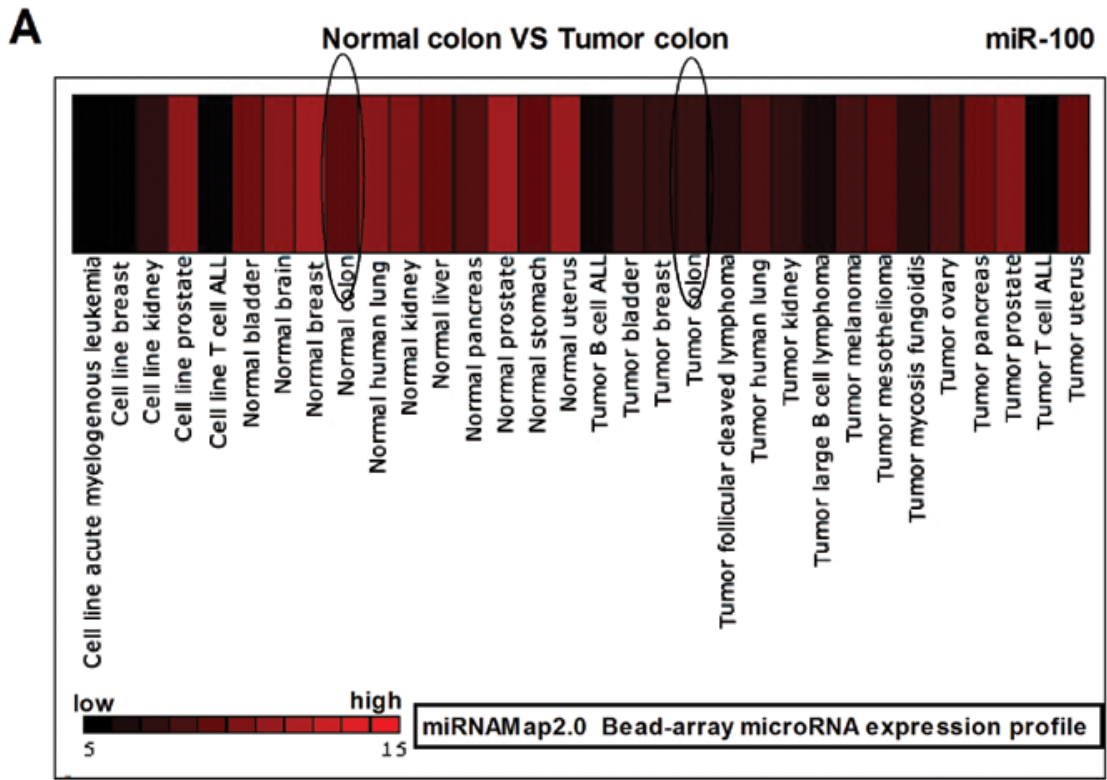

\section{B}

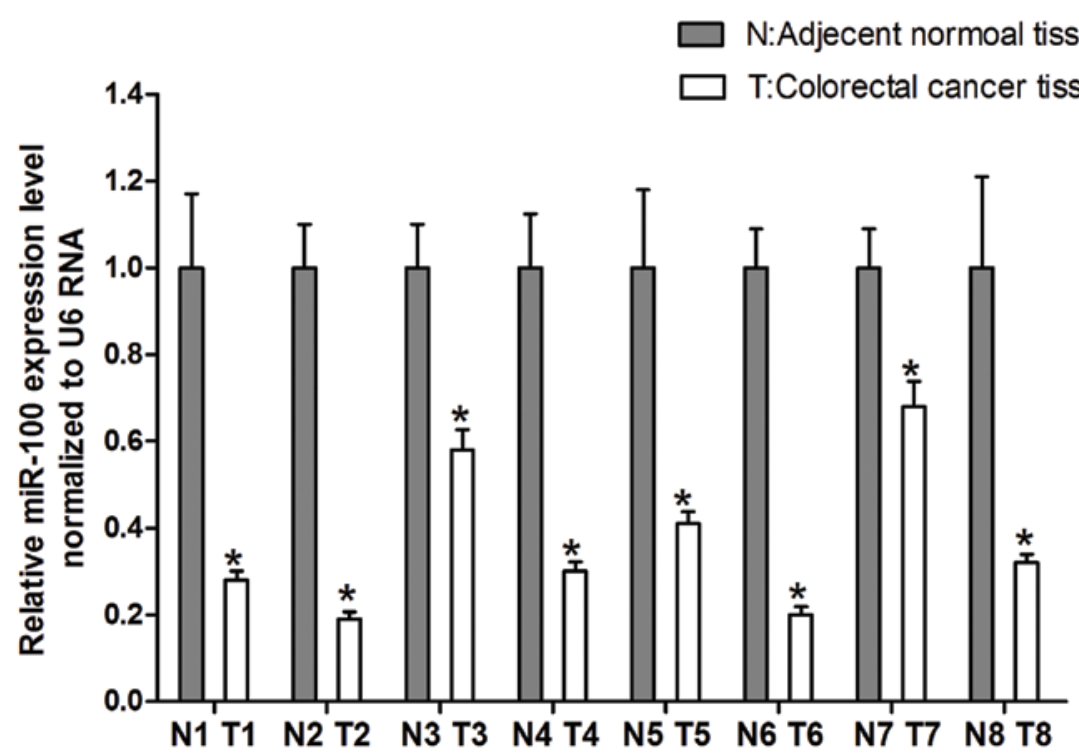

Figure 1. The expression of miR-100 is downregulated in colorectal cancer. (A) We searched the database of miR-100 expression in normal and tumor colon tissues by miRNAMap-2.0, and found that miR-100 was commonly downregulated in tumor human colon tissues compared to normal human colon tissues. (B) Quantitative analysis of the expression levels of miR-100 normalized to those of U6 by qRT-PCR. "P<0.05.

detection showed that overexpression of miR-100 decreased cell viability in SW620 cells lines at 48 h after transfection. The inhibition of miR-100 increased SW620 cell proliferation (Fig. 2B). Colony formation assays were used to further elucidate the effect of miR-100 on the growth of SW620 cells. The colony formation rate of SW620 cells transfected with miR-100 was reduced $\sim 65 \%$ over that of the control group (Fig. 2C). The opposite phenomenon was observed in SW620 cells transfected with anti-miR-100. These results revealed that miR-100 regulates the proliferation of human colorectal cancer SW620 cell lines in vitro. Moreover, to determine whether miR-100 is involved in tumorigenesis of colorectal cancer in vivo, we injected SW620 cells into the flanks of nude mice. One week after the injections, mice with compa- rably sized tumors were treated for 4 weeks with miR-NC and miR-100 mimics, and the tumor growth activity was measured. When tumors were harvested, the average weight of tumors derived from the miR-100 group was lower than that of the control group (Fig. 2D). These results were consistent with the effects of miR-100 in vitro and strongly suggest that miR-100 regulated proliferation of human colorectal cancer SW620 cells.

miR-100 regulates apoptosis and invasion of human colorectal cancer SW620 cells in vitro. To determine whether the SW620 cell growth regulation was attributed to apoptosis, we performed flow cytometric analysis of SW620 cells after transfection of miR-100 mimics, anti-miR-100 or their relative 
A

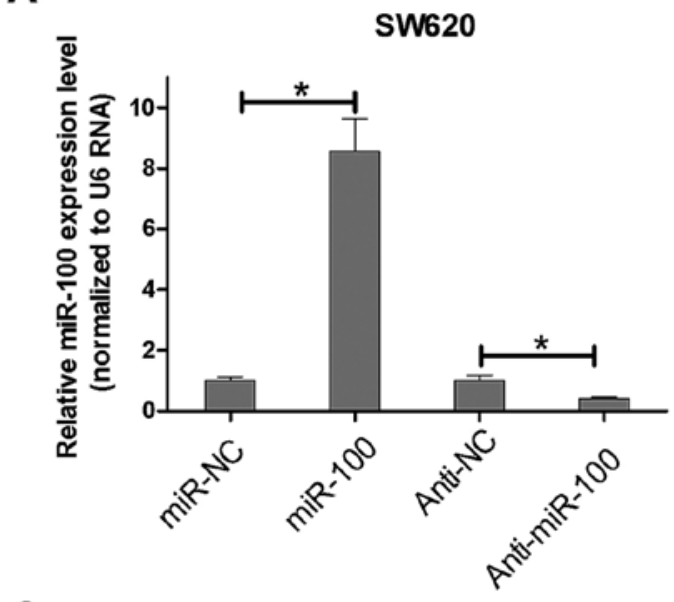

C

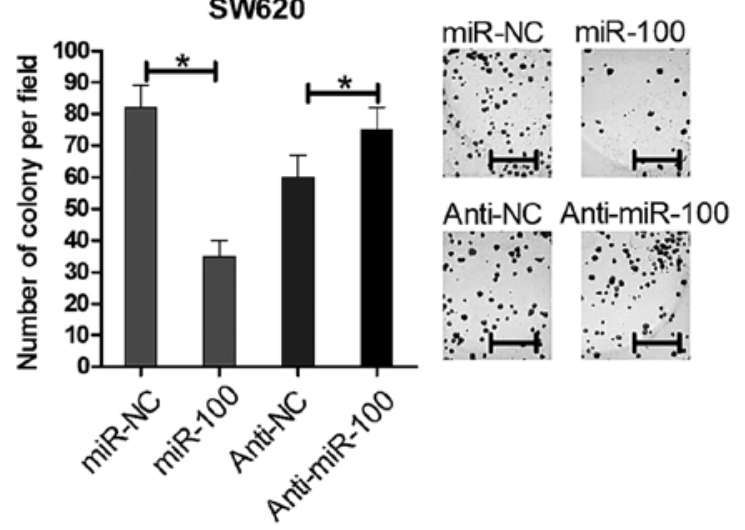

B

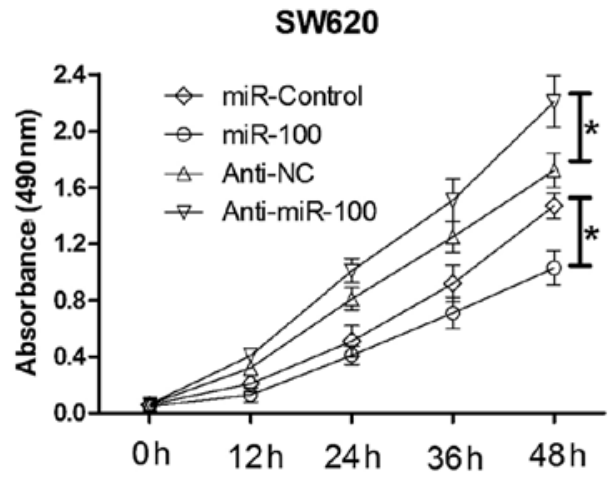

$\mathbf{D}$

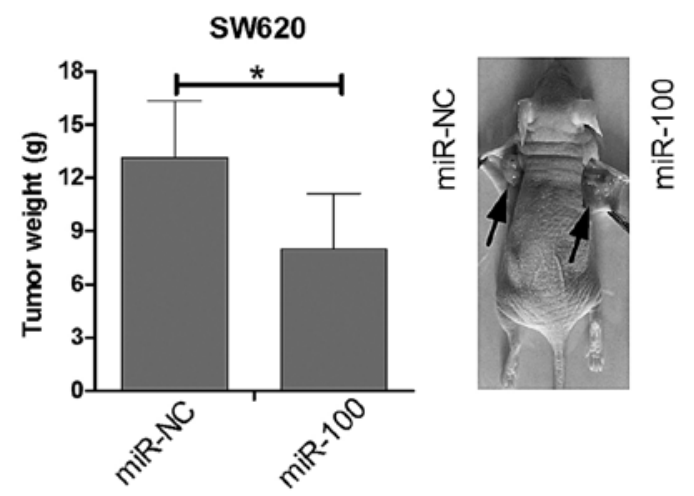

Figure 2. Effects of miR-100 on the cell growth of SW620 cells. (A) miR-100 expression level was analyzed by qRT-PCR. (B) MTT assay was performed to detect the effect of miR-100 mimics or anti-miR-100 mimics on the cell viability in SW620 cells with 12, 24, 36 and $48 \mathrm{~h}$ after transfection. (C) Colony formation assay was performed to detect the effect of miR-100 mimics or anti-miR-100 on cell growth in SW620 cells. The colonies were stained with crystal violet and then counted. Representative images are shown, and data are presented as the means \pm SD from three independent experiments. "P $<0.05$. Scale bar, $0.5 \mathrm{~cm}$. (D) Repression of growth of SW620 cells in nude mice by miR-100 treatment. The images show the difference in tumor weight between the miR-100 and control group $\left({ }^{*} \mathrm{P}<0.05\right)$.

controls. In miR-100 or anti-miR-100 transfected SW620 cells, the rates of early apoptosis/necrosis (D2 quadrant) were 20.3 and $19.0 \%$, while the rates in miR-control and anti-miR-100 control transfected cells were 9.6 and $25.2 \%$, respectively (Fig. 3A and B). These results suggest miR-100 induced while anti-miR-100 reduced apoptosis of SW620 cells.

To determine whether miR-100 could regulate invasion of SW620 cells, we performed Transwell invasion assays. As shown in Fig. C and D, SW620 cells transfected with miR-100 mimics displayed invasion ability inhibition when compared with the control group, while transfected with anti-miR-100 have the opposite effect. The above data indicated that miR-100 not only could regulate SW620 cell growth and apoptosis, but also impaired SW620 cell invasion.

$R A P 1 B$ directly targeted by miR-100 is inversely expressed with miR-100 in human colorectal cancer. Given that miR-100 has pivotal function in human colorectal cancer SW620 cells, the question how the miRNA exerts its role in colorectal cancer needs to be investigated. TargetScan prediction algorithm was used for computational screen of genes with complementary sites of miR-100 in their 3'-UTR. We found that RAP1B, a member of RAS oncogene family, was a putative target of
miR-100 in the top 100 predicted targets (http://www. targetscan.org/cgi-bin/targetscan/vert_61/targetscan.cgi? species $=$ Human $\&$ gid $=\&$ mir_c $=\&$ mir_sc $=m i R-99 a b / 100 \&$ mir_nc $\left.=\& \operatorname{mirg}=\& \operatorname{sortType}=\& a l l T x s=\& i n c l \_n c=100\right)$. To confirm this possibility, the miR-100 binding sequences present at the 3'-UTR of RAP1B mRNA (WT-3'-UTR), its mutant site (RAP1B-3'UTR-mut) were subcloned downstream of the luciferase reporter gene in pGL3 vector (Fig. 4A) and then co-transfected into SW620 cells. The relative luciferase activity of the reporter that contained wild-type 3'-UTR was decreased by $70 \%$ when miR-100 was co-transfected and it was increased by $28 \%$ when anti-miR-100 was co-transfected, but the luciferase activity of RAP1B-3'UTR-mut reporter was unaffected by simultaneous transfection of miR-100 or anti-miR-100. These results suggesting that miR-100 might suppress RAB14 expression through the putative binding site in its 3'-UTR.

Next, real-time PCR and western blot assays were performed to check whether miR-100 expression affects the expression of endogenous RAP1B at both transcriptional and translational levels. Consistent with the results of Luciferase report assay, the levels of RAB14 mRNA showed a significant decrease between miR-100 mimic-transfected SW620 cells and miR- 
A

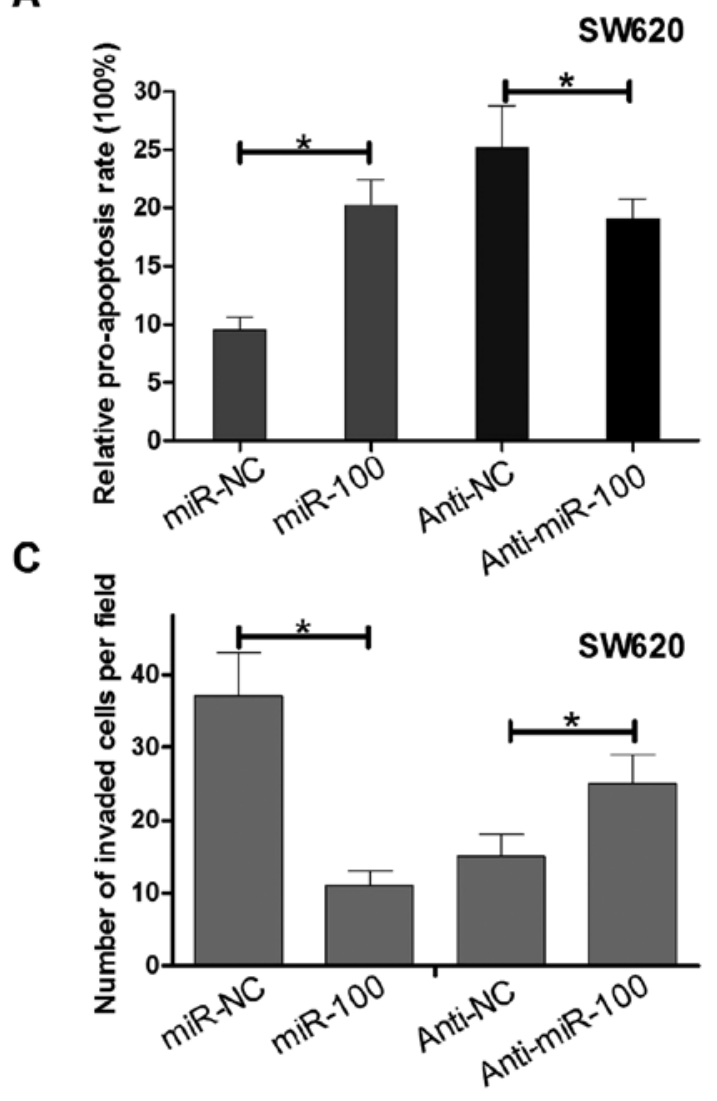

B

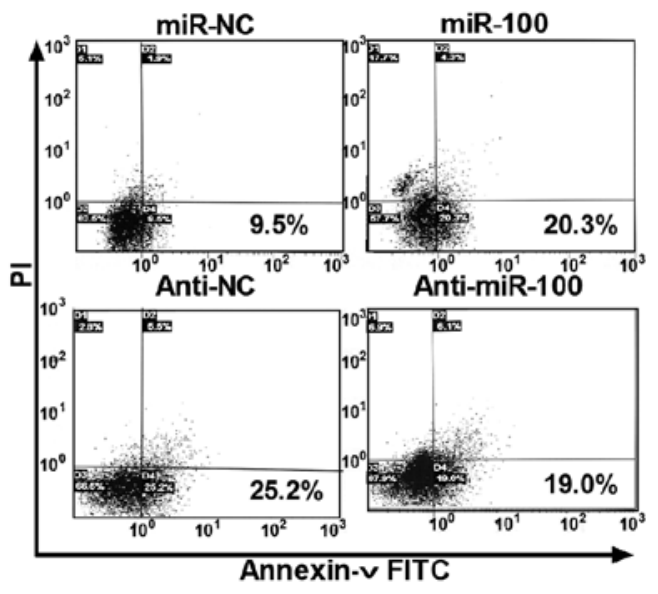

D

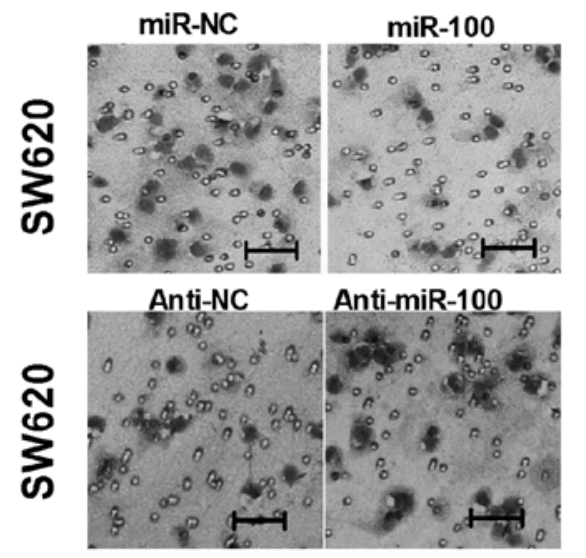

Figure 3. miR-100 regulates apoptosis and invasion of human colorectal cancer SW620 cells in vitro. (A) In the SW620 cells, apoptosis was evaluated by flow cytometry. The results revealed a marked increase in apoptosis in miR-100 mimic-transfected cells, while a marked decrease in apoptosis in anti-miR-100 mimic-transfected cells compared to the relative control groups. Representative flow cytometry report is shown (B). (C and D) The invasive activity of SW620 cells treated with miR-100 or anti-miR-100 was detected using a Transwell invasion assay. The number of cells traversing the membrane were determined by averaging nine random fields. The bars represent the mean \pm SD of three independent experiments ( $\mathrm{P}<0.05)$. Scale bar, $100 \mu \mathrm{m}$.

Table I. Primers and sequence in this study.

\begin{tabular}{lll}
\hline Name & Primer & \multicolumn{1}{c}{ Sequence } \\
\hline U6 & Forward & 5'-GTGCTCGCTTCGGCAGCACATATAC-3' \\
& Reverse & 5'-AAAAATATGGAACGCTCACGAATTTG-3' \\
GAPDH & Forward & 5'-ATGTCGTGGAGTCTACTGGC-3' \\
& Reverse & 5'-TGACCTTGCCCACAGCCTTG-3' \\
miR-100 & Forward & 5'-ACACTCCAGCTGGGAACCCGTAGATCCGAA-3' \\
& Reverse & 5'-CTCAACTGGTGTCGTGGAGTCGGCAATTCAGTTGAGCACAAGTT-3' \\
RAP1B & Forward & 5'-TTTATTCCATCACAGCACAGTCC-3' \\
& Reverse & 5'-TTTCTGTTAATTTGCCGCACTAGG-3' \\
miR-100 mimics & mi-100 & 2'-O-Me-AACCCGUAGAUCCGAACUUGUG \\
miR-100 inhibitor & Anti-sense miR-100 & 2'-O-Me-CACAAGUUCGGAUCUACGGGGG \\
\hline
\end{tabular}

control-transfected cells or between anti-miR-100 transfected SW620 cells and anti-miR-control transfected cells (data not shown). Moreover, western blot analysis showed that the level of RAP1B protein expression in miR-100 transfected SW620 cells was inhibited by $90 \%$ compared with that in miR-control transfected cells, while the level of RAP1B protein expression in anti-miR-100 transfected SW620 cells was upregulated by $45 \%$ compared with that in anti-miR-control transfected cells (Fig. 4C). Having identified RAP1B as a target of miR-100, we assessed the relationship of the expression of miR-100 and RAP1B in human colorectal cancer tissues. Fig. 4D shows the RAP1B mRNA was inversely expressed with miR-100 
A

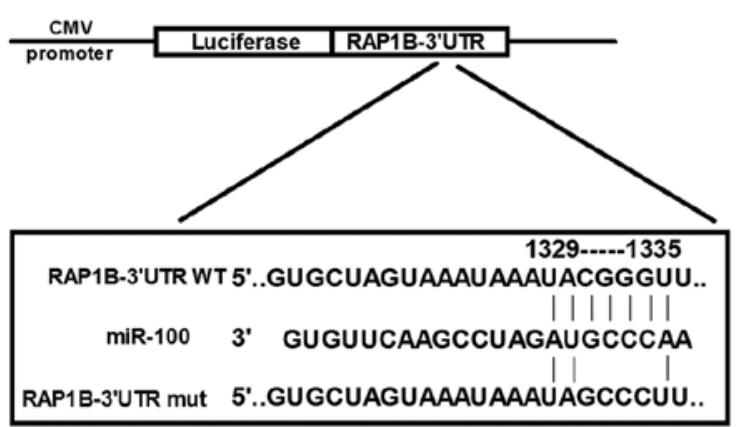

C

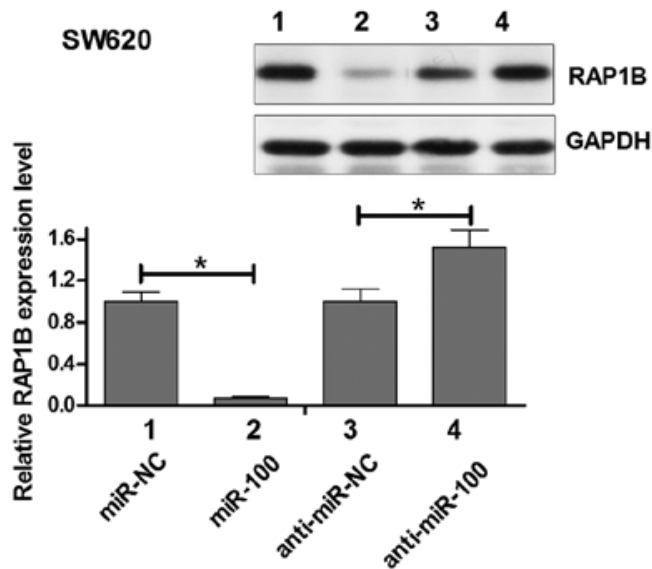

B

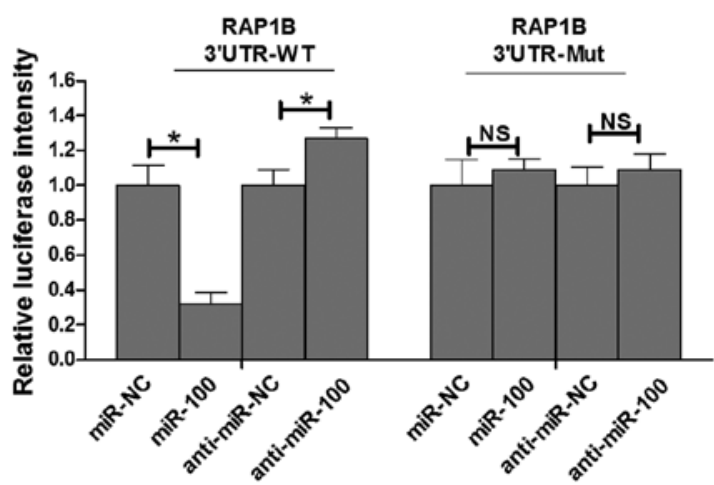

D

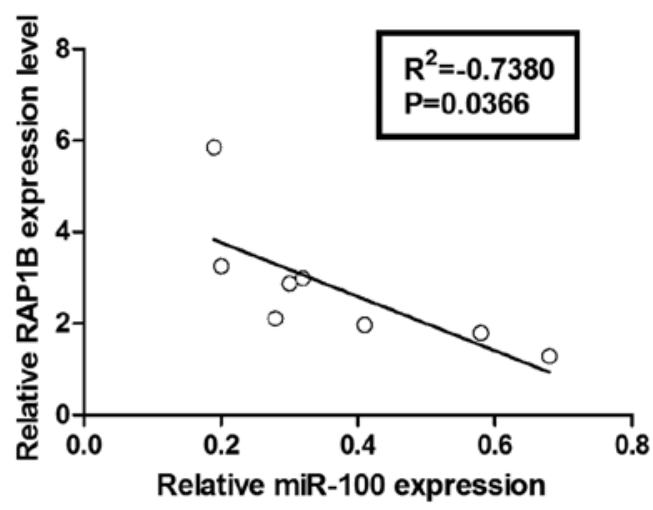

Figure 4. RAP1B is a potential target for miR-100. (A) The algorithms between miR-100 and the 3'-UTR of RAP1B and the mutant RAP1B 3'-UTR (four nucleotides within binding sites were mutated). (B) Luciferase reporter assay was performed to detect the effect of miR-100 and anti-miR-100 on the Luciferase intensity controlled by 3'-UTR of RAP1B ("P<0.05; NS, no significance). (C) Western blot was preformed to detect the effect of miR-100 mimics and antimiR-100 mimics on the protein levels of RAP1B. Representative images are shown, and data are presented as the means \pm SD from three independent experiments. $\mathrm{P}<0.01$. (D) qRT-PCR was performed to detect the relationship between the expression of miR-100 and RAP1B mRNA in colorectal cancer tissues. Pearson product-moment correlation coefficient was used to measure the correlation. $\mathrm{P}<0.05$ indicates the correlation is statistically significant $(\alpha=0.05)$.

\section{miRNAs/RAP1B axis in tumor cells}

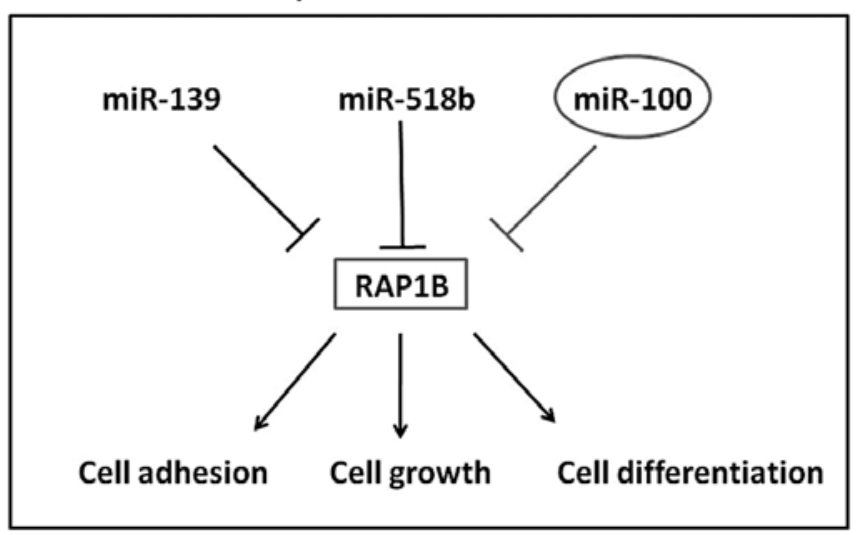

Figure 5. Schematic representation of the hypothetical molecular mechanism of miR-100/RAP1B axis regulating the proliferation, apoptosis and invasion of CRC cells.

in human colorectal cancer and statistically significant in the Pearson relation mean $\left(\mathrm{R}^{2}=-0.7380, \mathrm{P}=0.0366\right)$. These data indicated that miR-100 could negatively regulate RAP1B expression in human colorectal cancer SW620 cells by inter- action with the putative binding site in 3'-UTR of RAP1B mRNA, which may partly explained the miR-100 induced growth, apoptosis and invasion regulation mechanism of SW620 cells.

\section{Discussion}

It has been widely demonstrated that miRNAs regulate diverse biological processes, including tumorigenesis. The role of miR-100 is reported to be frequently downregulated in human cancer, such as acute lymphoblastic leukaemia, hepatocellular carcinoma, human esophageal squamous cell carcinoma, human bladder urothelial carcinoma, nonsmall cell lung cancer and breast cancer (9-14). However, its expression patterns in tumors are controversial. Wang et al reported that miR-100 overexpression strongly associates with advanced tumor progression and unfavorable clinical outcome of patients with renal cell carcinoma (RCC) (15). Herein, we focused on the regulation of miR-100 in colorectal cancer. First, we utilized the miR-map2.0 software to test the expression of miR-100 in normal colon tissues and tumor colon tissues. Next, we examined miR-100 expression in human colorectal cancer tissues and matched normal tissues by realtime RT-PCR assay. We discovered that the levels of miR-100 
were downregulated in tumor tissues, compared with the matched normal tissues in eight pairs of matched specimens. Therefore, we hypothesized that the downregulated miR-100 may function as a tumor suppressor gene in colorectal cancer, which was consistent with most of other human solid tumor types.

Using the MTT and colony formation assays to detect the effect of miR-100 on the growth capacity of colorectal cancer cell lines in vitro, we found that SW620 cells transfected with the miR-100 mimics exhibited decreased growth compared with the control cells while transfected with the miR-100 inhibitor exhibited increased growth. The in vivo study also demonstrated miR-100 could reduce SW620 cell proliferation. Thus, we inferred that miR-100 may be a growth inhibition factor in colorectal cancer.

To further reveal the exact role of miR-100 in colorectal cancer, we tested the effect of miR-100 on apoptosis and invasion by up- and downregulating the expression level of miR-100. The results showed that increased miR-100 induced apoptosis of SW620 cells, while decreased miR-100 inhibited apoptosis, suggesting that miR-100 suppressed the ability of SW620 cells to proliferate by inducing apoptosis in colorectal cancer. Since metastasis is an important feature of colorectal cancer, we examined the implication of miR-100 in SW620 cell invasion, and data showed that miR-100 had a negative effect on invasion suggesting that downregulation of miR-100 in colorectal cancer cells may play roles in the development of colorectal cancer through inhibiting cell proliferation, inducing apoptosis, and decreasing cell invasion. However, the inhibition of invasion ability by miR-100 in colorectal cancer should be confirmed with further in vivo experiments.

As we known, miRNAs are recognized as important regulators of gene expression, suppressing the expression of target genes through translational repression or degradation of a target transcript. We integrated bioinformatics-based predictions and the resulting candidate functions and found that the RAP1B gene had the highest recurrence rate as a potential target gene of miR-100. miRNAs are believed to bind partially to the homologous sequence of a target gene in the 3'-UTR. Accordingly, we constructed a luciferase reporter plasmid bearing the wild-type 3'-UTR of RAP1B mRNA for in vitro analysis. We found that inhibition or overexpression of miR-100 could significantly enhance or reduce luciferase expression, respectively. Furthermore, we constructed another luciferase reporter vector containing a mutated miR-100 'seed region' binding site, and no significant difference was detected when miR-100 was either overexpressed or inhibited. These results suggested that miR-100 can directly and negatively regulate RAP1B gene expression by binding to the 3'-UTR of RAP1B mRNA. It was suggested that highly expressed miRNAs can suppress target gene expression, whereas inhibition of an endogenous miRNA can protect mRNA targets from increased degradation. Accordingly, we utilized western blot assays to confirm the hypothesis that RAP1B was regulated by miR-100. We found that when miR-100 was blocked, RAP1B expression was enhanced, while miR-100 was overexpressed, RAP1B protein expression levels were reduced. Thus, we concluded that miR-100 negatively regulated the expression of RAP1B.
RAP1B was a member of the RAS-like small GTP-binding protein superfamily. Members of this family regulate multiple cellular processes including cell adhesion and growth and differentiation. RAP1B localizes to cellular membranes and has been shown to regulate integrin-mediated cell signaling. It also plays a role in regulating outside-in signaling in platelets (16-20). Recently, Rap1B was reported to be regulated by miR-139 in colorectal cancer and regulate by miR-518b in esophageal squamous cell carcinoma $(21,22)$. However, RAP1B induced mechanism of tumor cell malignant behavior is complex and multiple. Besides, it has been proposed that a single miRNA can target several genes and multiple miRNAs can target a single gene in a comprehensive manner $(23,24)$. In the present study, we identified that miR-100 negatively regulates the expression of RAP1B possibly supporting the hypothesis that the overexpression of RAP1B in colorectal cancer, at least partly, result from not only the underexpression of miR-139 but also miR-100. How many miRNAs participate in the RAP1B pathway on colorectal cancer need to be further elucidated.

Collectively, the present study provides evidence that miR-100 is downregulated in colorectal cancer tissues and that it functions as tumor suppressor inhibiting cell proliferation, invasion and promoting apoptosis. Moreover, its novel target gene, RAP1B, was identified and found to be negatively expressed with miR-100 in colorectal cancer tissues. Our results (Fig. 5) strongly support and supplement the mechanism of miR/RAP1B axis in tumor cell growth and invasion.

\section{Acknowledgements}

This study was financially supported by research grants from the National Natural Science Foundation of China (No. 1360337) and National Natural Science Foundation of Guangdong Province (No. S2012010009082).

\section{References}

1. Jemal A, Siegel R, Ward E, Murray T, Xu J and Thun MJ: Cancer statistics, 2007. CA Cancer J Clin 57: 43-66, 2007.

2. Cunningham D, Atkin W, Lenz HJ, et al: Colorectal cancer. Lancet 375: 1030-1047, 2010.

3. Cho KR and Vogelstein B: Genetic alterations in the adenoma - carcinoma sequence. Cancer 70 (Suppl 6): 1727-1731, 1992.

4. Fearon ER and Vogelstein B: A genetic model for colorectal tumorigenesis. Cell 61: 759-767, 1990.

5. Zhang J, Guo H, Zhang H, et al: Putative tumor suppressor miR-145 inhibits colon cancer cell growth by targeting oncogene Friend leukemia virus integration 1 gene. Cancer 117: 86-95, 2011.

6. Lin Y, Wu J, Chen H, et al: Cyclin-dependent kinase 4 is a novel target in micoRNA-195-mediated cell cycle arrest in bladder cancer cells. FEBS Lett 586: 442-447, 2012.

7. Hutvagner G and Zamore PD: A microRNA in a multipleturnover RNAi enzyme complex. Science 297: 2056-2060, 2002 .

8. Ma Y, Zhang P, Yang J, Liu Z, Yang Z and Qin H: Candidate microRNA biomarkers in human colorectal cancer: systematic review profiling studies and experimental validation. Int J Cancer 130: 2077-2087, 2012.

9. Liu J,Lu KH,Liu ZL, Sun M,De W and Wang ZX: MicroRNA-100 is a potential molecular marker of non-small cell lung cancer and functions as a tumor suppressor by targeting polo-like kinase 1 . BMC Cancer 12: 519, 2012. 
10. Li XJ, Luo XQ, Han BW, Duan FT, Wei PP and Chen YQ MicroR NA-100/99a, deregulated in acute lymphoblastic leukaemia, suppress proliferation and promote apoptosis by regulating the FKBP51 and IGF1R/mTOR signalling pathways. Br J Cancer 109: 2189-2198, 2013.

11. Sun J, Chen Z, Tan X, et al: MicroRNA-99a/100 promotes apoptosis by targeting mTOR in human esophageal squamous cell carcinoma. Med Oncol 30: 411, 2013.

12. Xu C, Zeng Q, Xu W, et al: miRNA-100 inhibits human bladder urothelial carcinogenesis by directly targeting mTOR. Mol Cancer Ther 12: 207-219, 2013.

13. Chen P, Zhao X and Ma L: Downregulation of microRNA-100 correlates with tumor progression and poor prognosis in hepatocellular carcinoma. Mol Cell Biochem 383: 49-58, 2013.

14. Gebeshuber CA and Martinez J: miR-100 suppresses IGF2 and inhibits breast tumorigenesis by interfering with proliferation and survival signaling. Oncogene 32: 3306-3310, 2013.

15. Wang G, Chen L, Meng J, Chen M, Zhuang L and Zhang L: Overexpression of microRNA-100 predicts an unfavorable prognosis in renal cell carcinoma. Int Urol Nephrol 45: 373-379, 2013.

16. Bernardi B, Guidetti GF, Campus F, et al: The small GTPase Raplb regulates the cross talk between platelet integrin alpha2beta1 and integrin alphaIIbbeta3. Blood 107: 2728-2735, 2006.
17. Carmona G, Gottig S, Orlandi A, et al: Role of the small GTPase Rap1 for integrin activity regulation in endothelial cells and angiogenesis. Blood 113: 488-497, 2009.

18. Matsuse M, Mitsutake N, Rogounovitch T, et al: Mutation analysis of RAP1 gene in papillary thyroid carcinomas. Endocr J 56: 161-164, 2009.

19. Edreira MM,Li S, Hochbaum D, et al: Phosphorylation-induced conformational changes in Raplb: allosteric effects on switch domains and effector loop. J Biol Chem 284: 27480-27486, 2009.

20. Wittchen ES, Aghajanian A and Burridge K: Isoform-specific differences between Rap1 A and Rap1B GTPases in the formation of endothelial cell junctions. Small GTPases 2: 65-76, 2011.

21. Zhang M, Zhou S, Zhang L, et al: miR-518b is down-regulated, and involved in cell proliferation and invasion by targeting Raplb in esophageal squamous cell carcinoma. FEBS Lett 586: 3508-3521, 2012.

22. Guo H, Hu X, Ge S, Qian G and Zhang J: Regulation of RAP1B by miR-139 suppresses human colorectal carcinoma cell proliferation. Int J Biochem Cell Biol 44: 1465-1472, 2012.

23. Hobert O: miRNAs play a tune. Cell 131: 22-24, 2007

24. Peter ME: Targeting of mRNAs by multiple miRNAs: the next step. Oncogene 29: 2161-2164, 2010. 